Mai 2003
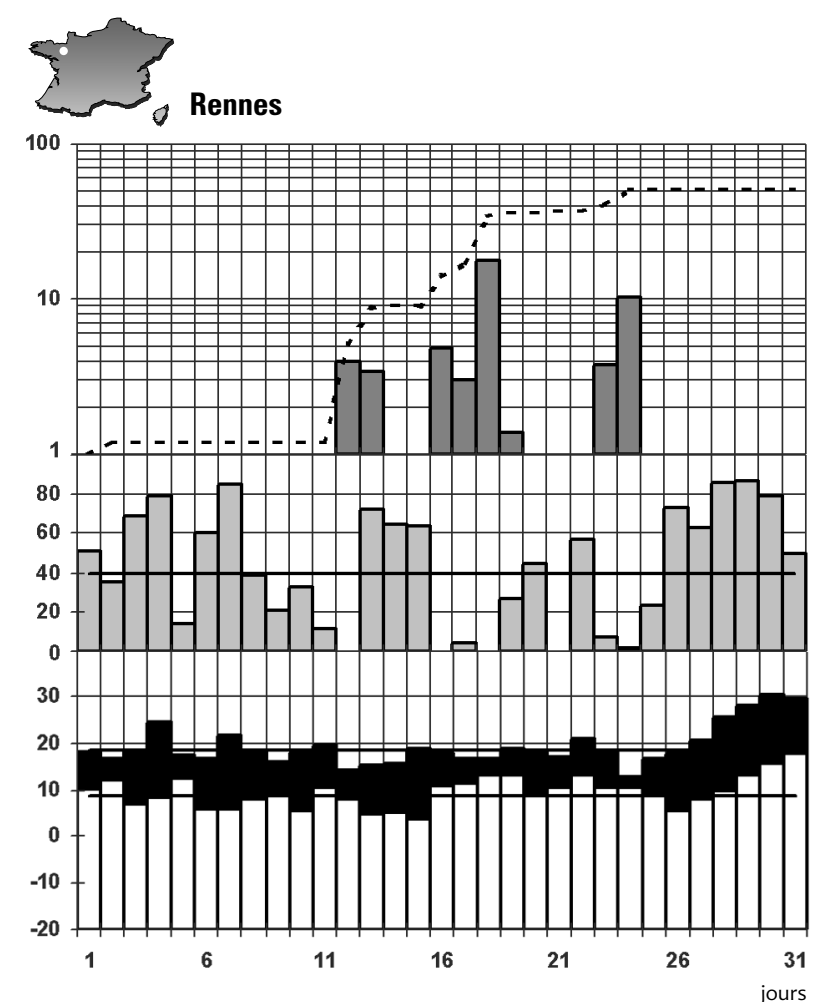

Total mensuel des précipitations : $80 \%$ de la normale

Fraction d'insolation moyenne : normale

Température moyenne : $>0,8^{\circ} \mathrm{C}$ à la normale
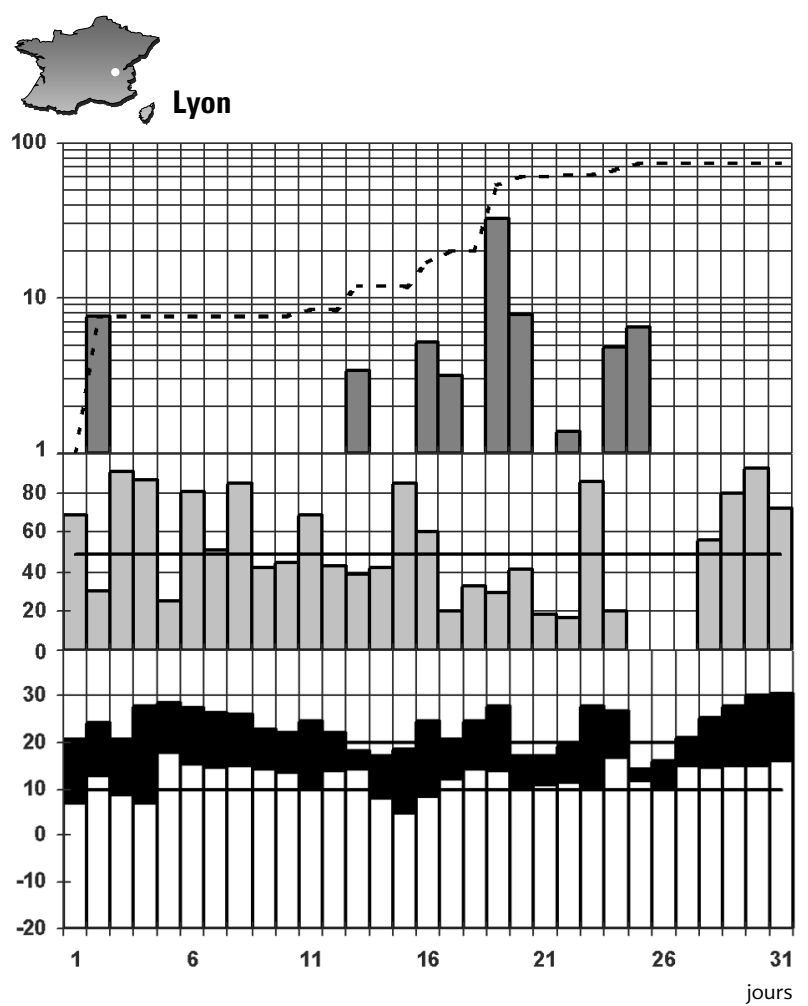

Total mensuel des précipitations : $90 \%$ de la normale

Fraction d'insolation moyenne : normale

Température moyenne : $>2,8^{\circ} \mathrm{C}$ à la normale

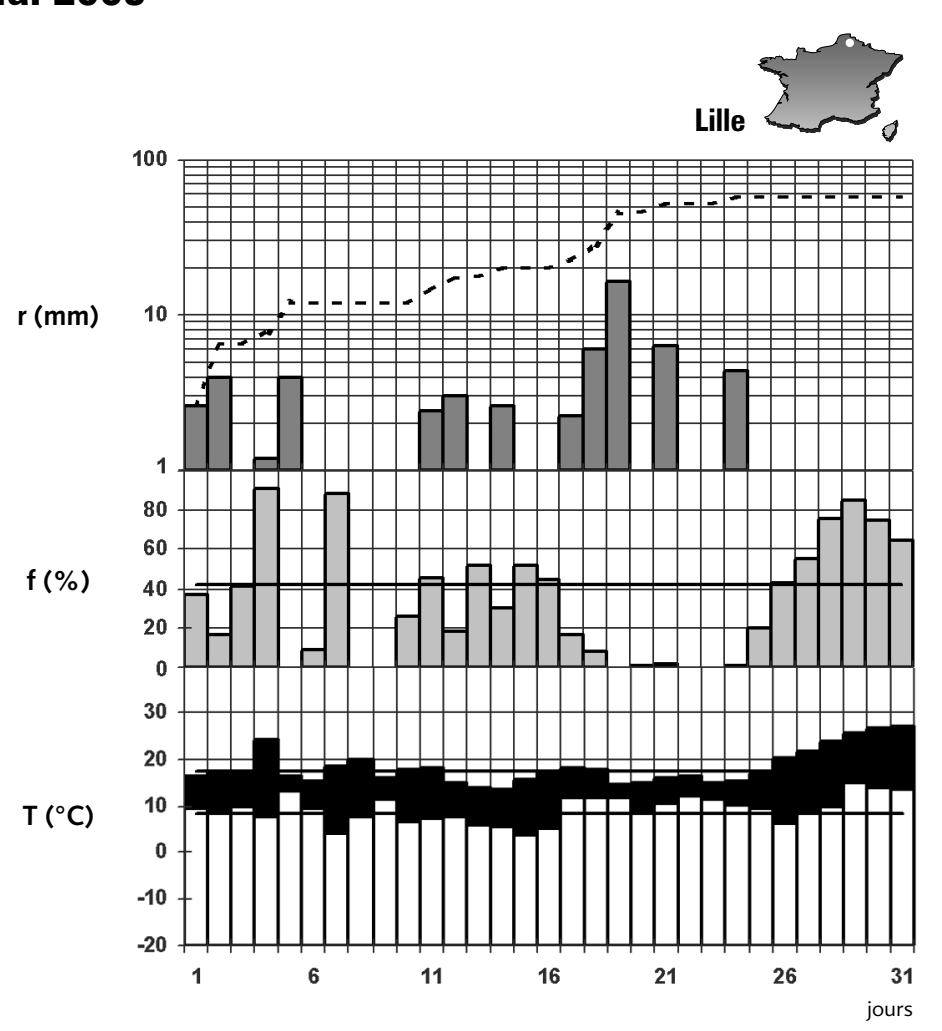

Total mensuel des précipitations : $90 \%$ de la normale

Fraction d'insolation moyenne : déficitaire

Température moyenne : $>0,7^{\circ} \mathrm{C}$ à la normale

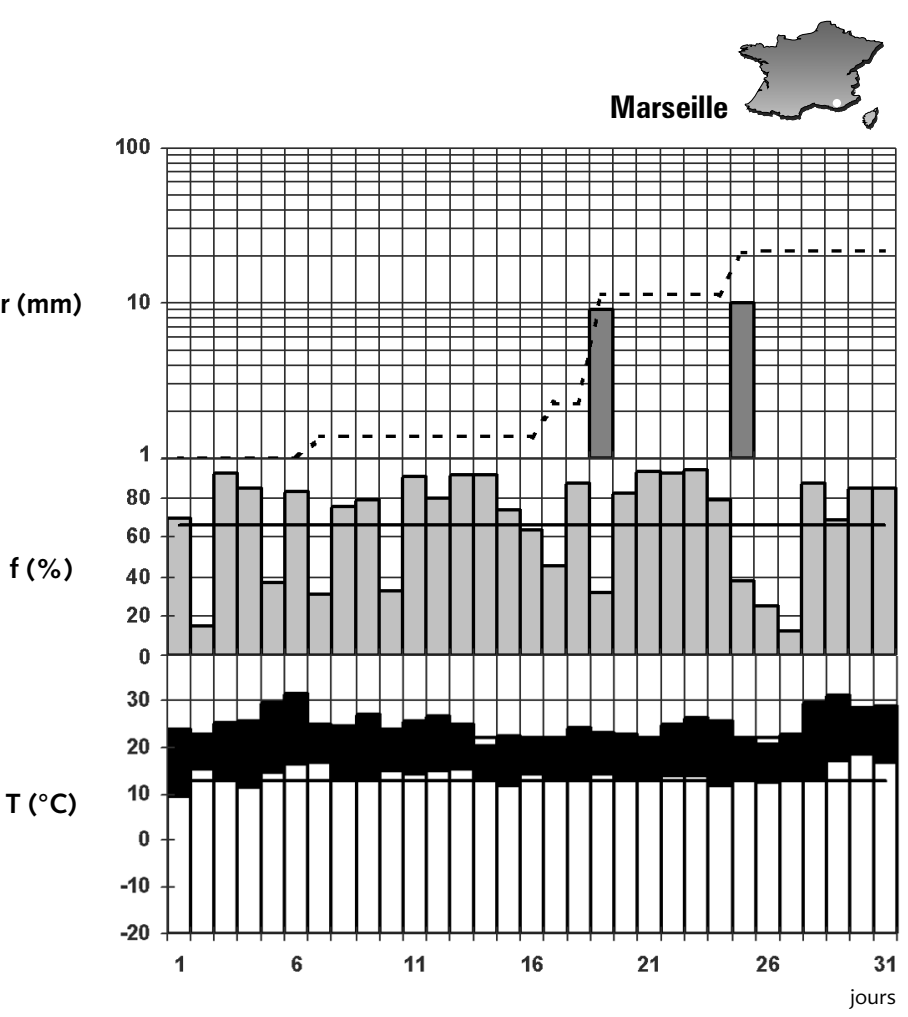

Total mensuel des précipitations : $50 \%$ de la normale

Fraction d'insolation moyenne : normale

Température moyenne : $>2,1^{\circ} \mathrm{C}$ à la normale 


\section{Mai 2003}
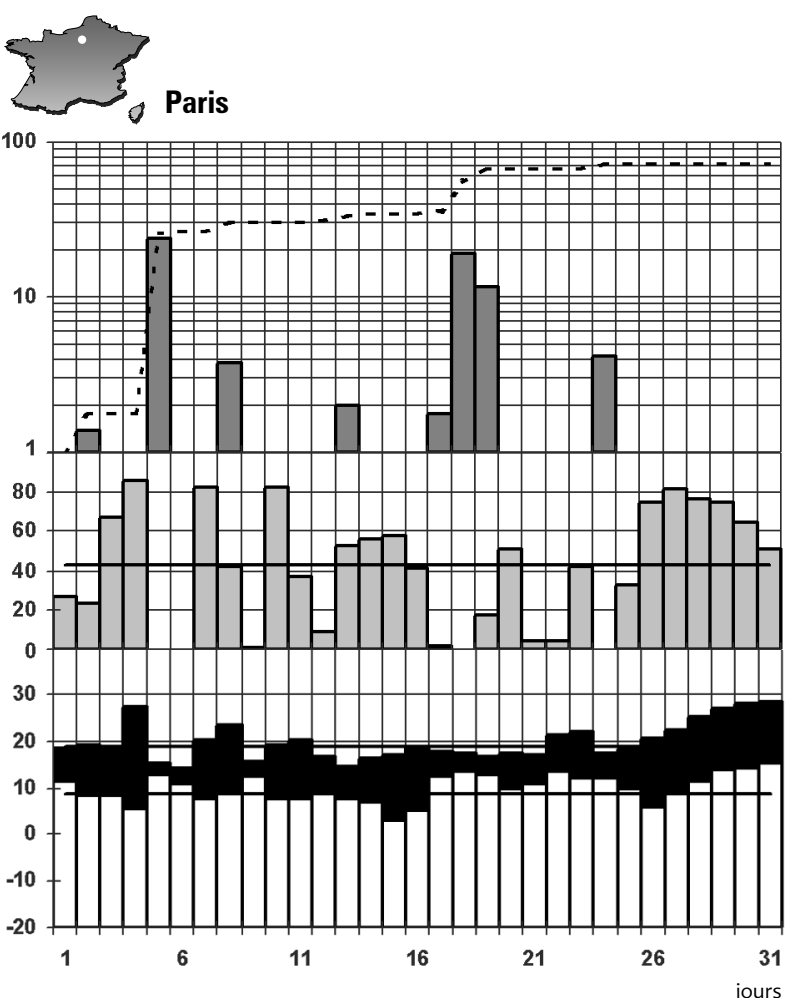

Total mensuel des précipitations : 1,2 fois la normale

Fraction d'insolation moyenne : normale

Température moyenne : > $1,1^{\circ} \mathrm{C}$ à la normale
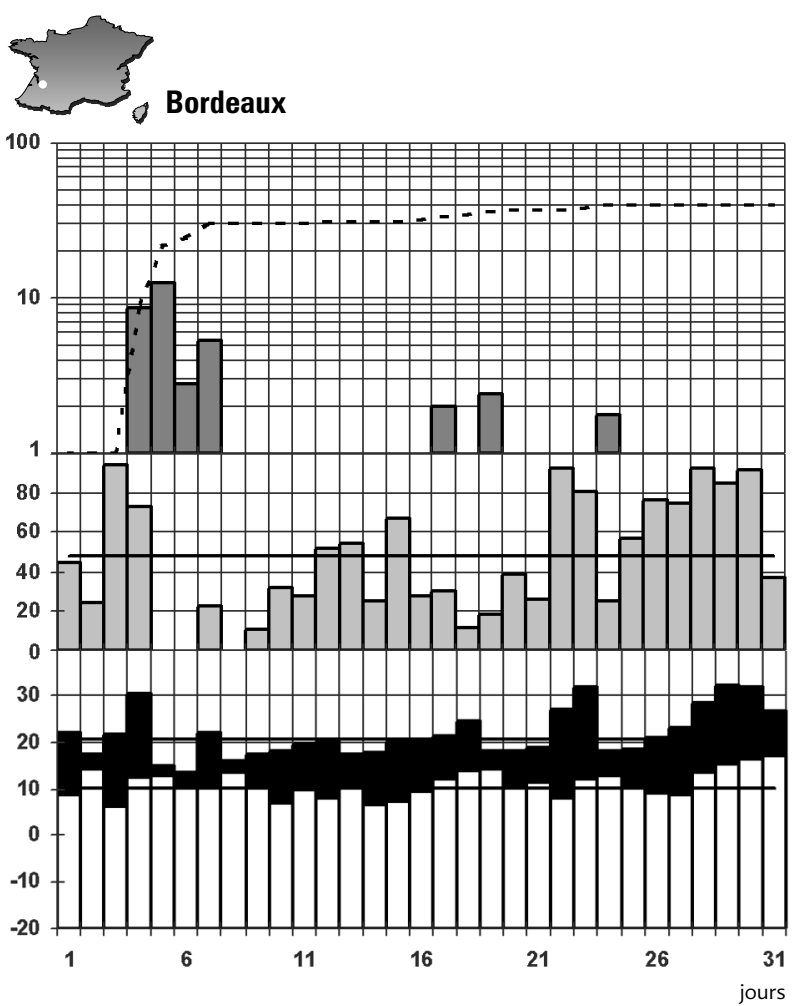

Total mensuel des précipitations : $50 \%$ de la normale

Fraction d'insolation moyenne : normale

Température moyenne : $>0,9{ }^{\circ} \mathrm{C}$ à la normale

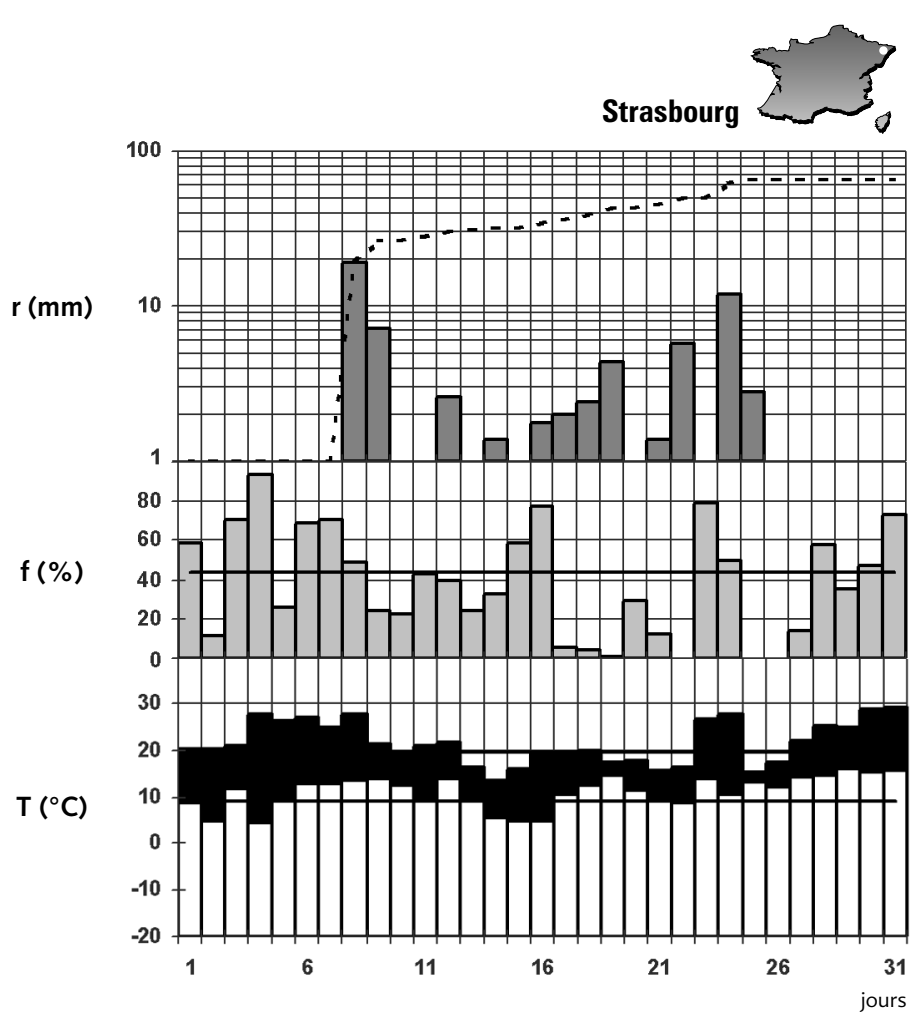

Total mensuel des précipitations : $80 \%$ de la normale Fraction d'insolation moyenne : légèrement déficitaire Température moyenne : > 2,0 ${ }^{\circ} \mathrm{C}$ à la normale

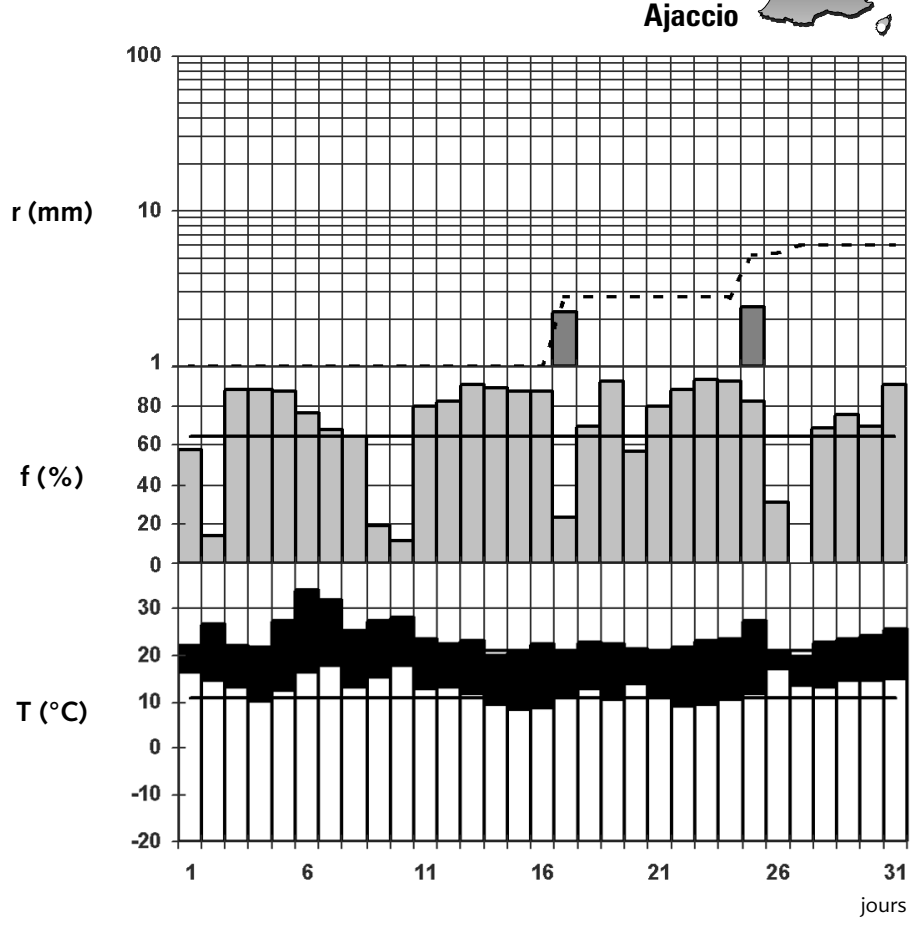

Total mensuel des précipitations : $10 \%$ de la normale

Fraction d'insolation moyenne : normale

Température moyenne : $>2,3^{\circ} \mathrm{C}$ à la normale

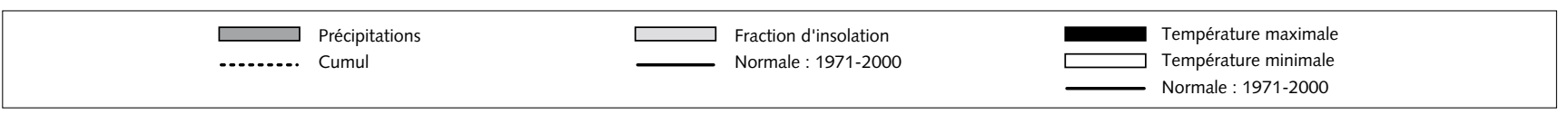




\section{Mai 2003}

Heures UTC sur tout le bulletin

\section{Périodes thermiques}

\section{Canicule en milieu de première décade ainsi qu'en fin de dernière.}

\section{Première décade}

Minimales. Le passage d'un système perturbé permet aux fins de nuit d'être douces le $1^{\text {er }}$ sur le tiers nord, sur l'extrême Sud-Est et la Corse, le 2 sur la majorité du pays à l'exception du NordEst : $16,4{ }^{\circ} \mathrm{C}$ [normale $9,3{ }^{\circ} \mathrm{C}$ ] à Ajaccio (Corse-du-Sud) le $1^{\text {er }} ; 13,7^{\circ} \mathrm{C}$ [normale $\left.8,1^{\circ} \mathrm{C}\right]$ à Sauternes (Gironde) le 2.

Le ciel dégagé des deux nuits suivantes fait baisser le mercure sur une grande partie de l'Hexagone : $2,3{ }^{\circ} \mathrm{C}$ [normale $7,9{ }^{\circ} \mathrm{C}$ ] à Mont-de-Marsan (Landes) le $3 ; 1,5{ }^{\circ} \mathrm{C}$ [normale 6,2 ${ }^{\circ} \mathrm{C}$ ] à Romilly (Aube) le 4.

En liaison avec une limite, une brutale remontée du thermomètre intervient le 5 sur le pays : $21,3{ }^{\circ} \mathrm{C}$ [normale $7,6{ }^{\circ} \mathrm{C}$ ] à Gémenos (Bouches-du-Rhône) et $19,9{ }^{\circ} \mathrm{C}$ [normale $6{ }^{\circ} \mathrm{C}$ ] au Cheylard (Ardèche) le 5.

Si les nuits restent très douces le 6 sur une grande moitié est, elles redeviennent normales sur la frange ouest ; les deux jours suivants, c'est une zone allant de la Bretagne à l'Artois qui enregistre les températures les plus fraîches, alors que, sur le reste du pays, elles sont par endroits bien au-delà des normales saisonnières : $0,2{ }^{\circ} \mathrm{C}$ [normale $5,1^{\circ} \mathrm{C}$ ] à Vire (Calvados) et $20,5{ }^{\circ} \mathrm{C}$ [normale $\left.8,9^{\circ} \mathrm{C}\right]$ au Luc (Var) le 7.

Le ciel couvert du 9 permet aux minimales d'être au-dessus des normales; le 10, elles se rafraîchissent sur l'Ouest : $13,1^{\circ} \mathrm{C}$ [normale $5,2^{\circ} \mathrm{C}$ ] à Roville-auxChênes (Vosges) le $9 ; 1,5^{\circ} \mathrm{C}$ [normale $\left.5,1^{\circ} \mathrm{C}\right]$ à Vire (Calvados) le 10.

Maximales. Elles sont globalement un peu supérieures aux valeurs saisonnières durant les trois premiers jours, le Midi enregistrant de chauds après-midi le 3 : $27,3{ }^{\circ} \mathrm{C}$ [normale $19,3{ }^{\circ} \mathrm{C}$ ] à Narbonne (Aude).

Grâce à une belle dorsale, les maximales sont exceptionnellement chaudes le 4 sur le pays, un tiers des stations dépassant sa moyenne décadaire de plus de $10^{\circ} \mathrm{C}$ : $26{ }^{\circ} \mathrm{C}$ [normale $13,4^{\circ} \mathrm{C}$ ] à Dieppe (Seine-Maritime), $30,4{ }^{\circ} \mathrm{C}$ à BordeauxMérignac (Gironde) [ancien record $30,1{ }^{\circ} \mathrm{C}$ ], $32{ }^{\circ} \mathrm{C}$ [normale $19,4^{\circ} \mathrm{C}$ ] à Dax (Landes) et $32,9{ }^{\circ} \mathrm{C}$ à Saint-Martin-deHinx (Landes).
Les 5 et 6 , elles sont encore très chaudes sur l'Est, mais déficitaires sur l'Ouest : $11,7^{\circ} \mathrm{C}$ [normale $18,8^{\circ} \mathrm{C}$ ] à ToulouseBlagnac (Haute-Garonne), $31{ }^{\circ} \mathrm{C}$ à Carpentras (Vaucluse) [ancien record $30,1^{\circ} \mathrm{C}$ et $33,8^{\circ} \mathrm{C}$ à Ajaccio (Corse-duSud) [ancien record $32,8^{\circ} \mathrm{C}$ ] le 6 .

Les côtes de la Manche ainsi qu' un petit puis un grand quart sud-ouest sont respectivement les seuls à être déficitaires les 7 et $8: 31,8^{\circ} \mathrm{C}$ [normale $19,5{ }^{\circ} \mathrm{C}$ ] à Ajaccio (Corse-du-Sud) le 7.

Une belle amplitude thermique existe encore le 9 entre l'Est excédentaire et l'Ouest déficitaire, mais sans commune mesure avec celles des 5 et 6 . Le 10 est une journée conforme aux normales.

\section{Deuxième décade}

Minimales. Les fins de nuit oscillent autour des valeurs de saison le 11. Les 12 et 13, l'amplitude thermique grandit, le 12 entre l'Est et l'Ouest, le 13 entre le Nord et le Sud : 4,7 ${ }^{\circ} \mathrm{C}$ [normale $\left.8,9^{\circ} \mathrm{C}\right]$ à Saint Nazaire-Montoir (LoireAtlantique) et $16,9^{\circ} \mathrm{C}$ [normale $11,2^{\circ} \mathrm{C}$ ] à Arles (Bouches-du-Rhône) le 13.

Une brutale chute du mercure se fait sentir le 14 et se poursuit par endroits le 15 sur l'ensemble du territoire ; les gelées réapparaissent : $-1,6^{\circ} \mathrm{C}$ à Auberive (Haute-Marne) le $14 ;-0,7{ }^{\circ} \mathrm{C}$ [normale $\left.7,3{ }^{\circ} \mathrm{C}\right]$ à Nevers-Marzy (Nièvre) et $-1,6^{\circ} \mathrm{C}$ à Pontarion (Creuse) le 15.

La douceur regagne difficilement du terrain le 16, un grand quart nord-est enregistrant encore des minimales fraîches pour la saison : $-1,6^{\circ} \mathrm{C}$ [normale $\left.6,6{ }^{\circ} \mathrm{C}\right]$ à Châtillon-sur-Seine (Côte-d'Or).

La forte nébulosité de la fin de la décade permet aux valeurs de remonter et cette fois de se retrouver souvent bien au-dessus des normales avec, du 17 au 19 , près de la totalité des stations qui enregistre des excédents : $13,1{ }^{\circ} \mathrm{C}$ [normale $6,7^{\circ} \mathrm{C}$ ] aux Riceys (Aube) le $18 ; 17,8^{\circ} \mathrm{C}$ au Cap-Corse (Haute-Corse) le 19.

Le 20 voit une légère baisse généralisée du thermomètre et marque le retour à des minimales habituelles pour la saison.

Maximales. C'est avec des valeurs agréables que débute la décade, les $25^{\circ} \mathrm{C}$ étant souvent franchis dans le Sud-Est : $26,7^{\circ} \mathrm{C}$ [normale $19,7^{\circ} \mathrm{C}$ ] à Saint-Jeanen-Royans (Drôme) le 11.

$\mathrm{Si}$, dans cette région, le mercure ne baisse que très peu le 12 , il en est tout autrement sur un grand quart nordouest : $10,9{ }^{\circ} \mathrm{C}$ [normale $15,5^{\circ} \mathrm{C}$ ] $a ̀$ Rostrenen (Côtes-d'Armor).

Les 13 et 14 , une majorité de postes enregistre des déficits ; seul le Midi méditerranéen échappe en partie à cette fraîcheur : $12,7^{\circ} \mathrm{C}$ [normale $\left.19,4^{\circ} \mathrm{C}\right]$ à Luxeuil (Haute-Saône) le 14. Il fait meilleur le 15 au sud du $45^{\mathrm{e}}$ parallèle ainsi que sur la frange atlantique. Si le 16 la douceur concerne une grande partie du pays, les trois jours suivants, une amplitude thermique marquée apparait entre le Nord et le Sud, ce dernier enregistrant, à nouveau, souvent des valeurs supérieures à $25{ }^{\circ} \mathrm{C}: 28,6{ }^{\circ} \mathrm{C}$ [normale $20,6{ }^{\circ} \mathrm{C}$ ] Dax (Landes) le $18 ; 29,1^{\circ} \mathrm{C}$ [normale $\left.19,7^{\circ} \mathrm{C}\right]$ à Saint-Jean-en-Royans (Drôme) et $31,3{ }^{\circ} \mathrm{C}$ à Sainte-Marie-deCuines (Savoie) le 19.

\section{Troisième décade}

Minimales. Si les nuits sont globalement de saison jusqu'au 24, on enregistre toutefois des contrastes thermiques notables ; le 22, on relève des excédents du Massif central aux côtes de la Manche, alors que, sur un petit quart sud-ouest, les stations sont en déficit ; les 23 et 24 , le quart nord-est dans sa majorité profite de températures bien plus douces par rapport aux normales que le reste du pays : $5,3{ }^{\circ} \mathrm{C}$ [normale $\left.10{ }^{\circ} \mathrm{C}\right]$ à Mont-de-Marsan (Landes) et $13,8{ }^{\circ} \mathrm{C}$ [normale $7,3{ }^{\circ} \mathrm{C}$ ] à L'OudonLieury (Calvados) le $22 ; 14,2{ }^{\circ} \mathrm{C}$ [normale $8,3{ }^{\circ} \mathrm{C}$ ] à Nancy-Ochey (Meurtheet-Moselle) le 24.

Du 25 au 27, le thermomètre chute, de manière plus sensible dans l'Ouest en raison d'un bon rayonnement nocturne : $1,8{ }^{\circ} \mathrm{C}$ [normale $7,5{ }^{\circ} \mathrm{C}$ ] à Vire (Calvados) le 26.

La tendance est à une légère remontée du mercure le 28 , qui se confirme et s'accentue les trois jours suivants où plus aucune station n'est déficitaire : $19{ }^{\circ} \mathrm{C}$ [normale $9,7^{\circ} \mathrm{C}$ ] au Cap-de-laHève (Seine-Maritime) le $30 ; 19{ }^{\circ} \mathrm{C}$ [normale $7,3{ }^{\circ} \mathrm{C}$ ] à Pré-en-Pail (Mayenne) le 31.

Maximales. Cette décade contrastée débute par une journée fraîche avec une majorité de stations enregistrant un déficit de plus de deux degrés. Le ciel qui s'éclaircit le 22 sur le Sud et surtout le 23, à l'exception des côtes de la Manche, permet aux maximales de grimper en flèche, principalement dans le grand Sud-Ouest : $28,9{ }^{\circ} \mathrm{C}$ à Cham-bon-surVoueize (Creuse) [ancien record 28,5 ${ }^{\circ} \mathrm{C}$ ] et $33,3{ }^{\circ} \mathrm{C}$ [normale $20,9{ }^{\circ} \mathrm{C}$ ] à Sauternes (Gironde) le 23.

La fraîcheur gagne ensuite le territoire par l'ouest et si, le 24, l'est de la France enregistre encore de chaudes valeurs, les 25 et 26 , c'est tout le pays qui subit la baisse du mercure : $14,2{ }^{\circ} \mathrm{C}$ [normale $21,6^{\circ} \mathrm{C}$ ] à Voutezac (Corrèze) et 28,8 ${ }^{\circ} \mathrm{C}$ [normale $\left.20,1{ }^{\circ} \mathrm{C}\right]$ à Saint-Jean-en-Royans 
(Drôme) le $24 ; 13,5{ }^{\circ} \mathrm{C}$ [normale $21,2{ }^{\circ} \mathrm{C}$ ] à Carcassonne (Aude) le 26. Les températures remontent à un niveau de saison le 27 et, grâce à un soleil très présent, elles deviennent caniculaires du 28 au $31: 34{ }^{\circ} \mathrm{C}$ [normale 20,8 ${ }^{\circ} \mathrm{Cl}$ à Bazas (Gironde) et $35^{\circ} \mathrm{C}$ à Thouars (Deux-Sèvres) le 30 ; $33,7^{\circ} \mathrm{C}$ [normale $20,2^{\circ} \mathrm{C}$ ] à Glénay (Deux-Sèvres) le 31.

\section{Périodes pluviométriques}

\section{Quelques violents épisodes orageux.}

\section{Première décade}

Une perturbation intéresse le pays en ces deux premiers jours du mois. De faibles pluies tombent sur le littoral du Finistère au Nord, tandis qu'un ciel de marge donne quelques gouttes en soirée le $1^{\text {er }}$ sur le SudEst ; le lendemain, la limite passe sur la moitié nord avec des orages noyés dans la masse et des orages d'évolution diurne éclatent sur les Alpes : 10,8 mm à PleyberChrist (Finistère) le $1^{\text {er }}$; 28,4 mm à Embrun (Hautes-Alpes) le 2.

Après une journée du 3 où règne un temps sec et bien ensoleillé, une nouvelle perturbation ondule lentement sur le pays jusqu'au 6 dans un flux de sud. La dégradation s'amorce dans la nuit du 4 au 5, la limite pluvio-instable se dessine le 5 de l'ouest des Pyrénées aux Ardennes ; les pluies persistent le 6 sensiblement le long du même axe, alors que la perturbation se frontolyse. Les précipitations s'amenuisent surtout dans la partie nord : 30,6 $\mathrm{mm}$ à Royan (Charente-Maritime) le 4 ; $20 \mathrm{~mm}$ entre 18 h 00 et 24 h 00 à Gourdon (Lot), 43 mm à Aubigny-sur-Nère (Cher), 56 mm à Pierre-Buffière (Haute-Vienne) et $57,2 \mathrm{~mm}$ à Lannemezan (HautesPyrénées) le 5 ; 33,6 mm à la Pointe-deSocoa (Pyrénées-Atlantiques) et 39,4 mm Eymoutiers (Haute-Vienne) le 6.

Des averses se produisent le 7 sur un grand quart sud-ouest sous une zone de convergence et une activité orageuse se développe le 8 de la Gironde aux Pays de la Loire ainsi que de la Franche-Comté au nord de l'Alsace : 31,5 mm à Segonzac (Charente) le 7 ; 30,7 mm à Loudun (Vienne) et 47,8 mm à Hegeney (Bas-Rhin) le 8.

Une nouvelle onde progresse lentement vers l'est le 9. Des résidus traînent encore sur la façade est le 10 où ils sont confrontés à des remontées chaudes temporairement orageuses, tandis que de petites pluies touchent la pointe bretonne : 14,2 $\mathrm{mm}$ à SaintChristol(Vaucluse) le 10.

\section{Deuxième décade}

S'étendant même jusqu'au Massif central, l'instabilité sur l'Est persiste le 11 avec des averses orageuses : 13,6 $\mathrm{mm}$ à Vichy-Charmeil (Allier) avec de l'orage et 42,6 mm à Embrun (Hautes-Alpes) dont 15,8 mm entre $18 \mathrm{~h} 00$ et $19 \mathrm{~h} 00$.

Les ondées liées à une perturbation sur le nord du pays descendent le 12 du Centre au Nord-Est dans la traîne associée ; à l'arrière, un corps pluvio-instable se dessine le long de la Manche : $11 \mathrm{~mm}$ à Amiens-Glisy (Somme) le $11 ; 14,4 \mathrm{~mm}$ au Gast (Calvados) le 12.

L'établissement d'une masse d'air froid et instable génère le 13 des averses localement orageuses sur le nord et l'est de la Loire ; cette instabilité régresse le 14 vers le NordEst : 15,8 mm à Maîche (Doubs) le 13.

De petites averses se produisent encore le 15 sur le Nord et le corps pluvieux de l'onde arrivée dans la nuit sur la pointe bretonne se situe le 16 sur un petit quart nord-ouest ; par ailleurs, des averses se produisent du Massif central au Nord-Est sous des nuages de l'étage moyen et une évolution orageuse se propage rapidement du Sud-Ouest aux Cévennes : 9,8 $\mathrm{mm}$ à Brest-Guipavas (Finistère) et $31 \mathrm{~mm}$ à Durban-Corbières (Aude) le 16.

Tandis que la perturbation évacue le pays, une nouvelle arrive dans un flux d'ouest le 17. Des averses localement orageuses affectent le nord du $45^{\mathrm{e}}$ parallèle : 11,8 mm à Flers (Orne) entre $17 \mathrm{~h} 30$ et $18 \mathrm{~h} 30 ; 26,8 \mathrm{~mm}$ à Belfahy (Haute-Saône).

Une zone de pluies soutenues traverse rapidement le pays du sud de la Bretagne au Nord-Est le 18, puis, le 19, une limite active passe sur le pays accompagnée d'un dynamisme marqué : 37,4 $\mathrm{mm}$ à Rueil-la-Gadelière (Eure-et-Loir) et $40 \mathrm{~mm}$ à Palluau (Vendée) le $18 ; 20 \mathrm{~mm}$ au Pré-en-Pail (Mayenne) entre 7 h 30 et $10 \mathrm{~h}$ 30, 32,5 mm à Vivès (PyrénéesOrientales) et 37,8 mm Saint-Laurent-enGrandvaux (Jura) le 19.

La traîne est présente le 20 de la Gironde à la Franche-Comté et une nouvelle perturbation aborde la Bretagne : 20,5 $\mathrm{mm}$ à Burl (Ain).

\section{Troisième décade}

L'instabilité résiduelle concerne le NordEst le 21 et une limite chaude traverse la moitié nord du pays les 21 et 22 ; les restes pluvieux se situent sur le Nord-Est dans la matinée du $22: 13,8 \mathrm{~mm}$ à Lormes (Nièvre) le 21 ; 13,4 mm à Giromagny (Territoire-de-Belfort) le 22.

Un front froid s'enfonce le 23 par les côtes de la Manche. Prenant une composante très méridienne le 24 , il se réactive sur la majeure partie du pays, n'épargnant que le Sud-Est. Il poursuit son lent décalage vers l'est le 25 , des cellules orageuses se déve- loppant dans le Sud-Est : $19 \mathrm{~mm}$ à Valognes (Manche) le $23 ; 27,4$ mm à L'Oudon-Lieury (Calvados) et 38,4 mm à Castelnaudary (Aude) le 24 ; 26,4 mm à Saint-Vallier-de-Thiey (Alpes-Maritimes) et 34,6 mm à Yssingeaux (Haute-Loire) le 25. $\mathrm{Si}$ la perturbation ne présente plus d'activité dans sa partie nord le 26, dans le Sud, en revanche, elle est réactivée par une goutte froide méditerranéenne : 24,5 $\mathrm{mm}$ à Vivès (Pyrénées-Orientales) et 35,4 mm à Menton (Alpes-Maritimes) le 26.

Une petite évolution orageuse persiste le 27 sur l'extrême Sud-Est et le Corse ; mais, le 28 , de violents orages éclatent à proximité des reliefs de l'Est : $14 \mathrm{~mm}$ à Obernai (Bas-Rhin) et 56,4 mm à BâleMulhouse (Haut-Rhin) avec de l'orage, dont 54,6 mm entre $16 \mathrm{~h} 00$ et $17 \mathrm{~h} 00$; non loin de là, à Blotzheim, de nombreuses caves sont inondées par des torrents de boue.

Avec le décalage d'une petite goutte froide du Nord-Est vers le sud des Alpes, la convection et une forte dégradation orageuse se produisent sur ces massifs le 29 : 56,9 $\mathrm{mm}$ à La Grand-Combe (Gard), 60,2 mm à Digne-les-Bains (Alpes-deHaute-Provence) et 62,5 $\mathrm{mm}$ à Montfortsur-Argens (Var).

Les orages persistent le 30, mais diminuent très sensiblement en intensité ; la masse d'air très chaude est très fortement instable le 31, notamment sur le NordOuest : 19,6 mm à Saint-Michell'Observatoire (Alpes-de-Haute-Provence) le 30 ; 15,2 mm entre $15 \mathrm{~h} 36$ et 16 h 18 à Laval-Entrammes (Mayenne), 23,2 mm à Ticheville (Orne) et 30,4 mm à Octeville-sur-Mer (Seine-Maritime) le 31.

\section{La température}

La température moyenne du mois varie de $11,7{ }^{\circ} \mathrm{C}$ à Kerpert (Côtes-d'Armor) à 20,3 ${ }^{\circ} \mathrm{C}$ au Cap-Corse (Haute-Corse). L'écart à la normale varie d'un déficit de $1,1^{\circ} \mathrm{C}$ à Quimperlé (Finistère) à un excédent de $2,9^{\circ} \mathrm{C}$ au Cap-Corse (HauteCorse).

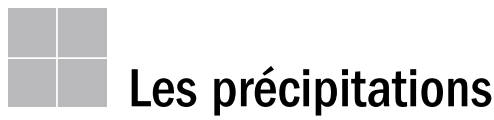

Le cumul mensuel varie de $0,4 \mathrm{~mm}$ à Saint-Jean-de-Maurienne (Savoie) à 135,2 mm à Saint-Léger-la-Montagne (Haute-Vienne). 
Le rapport à la normale varie de moins de $1 \%$ à Saint-Jean-de-Maurienne (Savoie) à $181 \%$ à Charleville-Mézières (Ardennes).

Le nombre de jours avec précipitations supérieures ou égales à $1 \mathrm{~mm}$ varie de 1 à Bormes-les-Mimosas (Var) à 17 à Lucelle (Haut-Rhin).

L'écart à la normale varie d'un déficit de 10,2 jours à Belcaire (Aude) à un excédent de 6,6 jours à Bléneau (Yonne).

\section{L'ensoleillement}

La durée d'insolation du mois varie de moins de 145 heures à Rostrenen (Côtes-d'Armor) à près de 312 heures à Carpentras (Vaucluse).

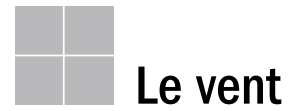

Un mois dans l'ensemble peu venté.

Associées à une perturbation qui arrive par le Nord-Ouest, de fortes rafales sont enregistrées en ce début de mois, principalement le 2 sur l'extrême nord du pays : $90 \mathrm{~km} / \mathrm{h}$ à la pointe de la Hague
(Manche) le $1^{e r} ; 101 \mathrm{~km} / \mathrm{h}$ à MetzFrescaty (Moselle), $104 \mathrm{~km} / \mathrm{h}$ à Brignogan (Finistère), $104 \mathrm{~km} / \mathrm{h}$ à Porten-Bessin (Calvados), $104 \mathrm{~km} / \mathrm{h}$ à SaintArnoult (Oise), $112 \mathrm{~km} / \mathrm{h}$ à la pointe de la Hague (Manche) et $122 \mathrm{~km} / \mathrm{h}$ au Cap-dela-Hève (Seine-Maritime) le 2.

Si le vent s'essouffle progressivement sur les côtes de la Manche le 3, un épisode de vent d'autan se déroule du 4 au 5 ; le 5, un flux de sud - sud-est concerne également très localement quelques régions au passage d'une limite : $101 \mathrm{~km} / \mathrm{h}$ et $94 \mathrm{~km} / \mathrm{h}$ à Labruguière (Tarn) les 4 et 5 ; $94 \mathrm{~km} / \mathrm{h}$ à Marignane (Bouches-du-Rhône) et $101 \mathrm{~km} / \mathrm{h}$ à Rouvroy-les-Merles (Oise) le 5.

La tramontane fait une courte apparition le 6, alors que, des Bouches-du-Rhône à la frontière italienne, c'est un vent d'est qui souffle ; le flux se montre également sensible sur les Pyrénées-Atlantiques : $86 \mathrm{~km} / \mathrm{h}$ à Trets (Bouches-du-Rhône), $94 \mathrm{~km} / \mathrm{h}$ à la Pointe-de-Socoa (Pyrénées-Atlantiques) et $97 \mathrm{~km} / \mathrm{h}$ à Leucate (Aude).

Rafales de secteur est à sud-est sont au menu du 7, à l'est du delta du Rhône ainsi que du Médoc à l'Aunis : $94 \mathrm{~km} / \mathrm{h}$ à Saint-Gervais (Gironde) et $112 \mathrm{~km} / \mathrm{h}$ à Grand-Courrent (Var).

Il faut attendre le 11 pour enregistrer à nouveau un coup de vent supérieur à $80 \mathrm{~km} / \mathrm{h}$ : $97 \mathrm{~km} / \mathrm{h}$ à Cambrai-Épinoy (Pas-de-Calais) sous orage.

Une activité instable sur le nord de la France engendre les 13 et 14 quelques fortes rafales ; mistral, tramontane et vent violent d'ouest à sud-ouest en Corse sont également présents : $112 \mathrm{~km} / \mathrm{h}$ à Dosnon (Aube), $112 \mathrm{~km} / \mathrm{h}$ à Leucate (Aude) et $115 \mathrm{~km} / \mathrm{h}$ au Cap-Corse (Haute-Corse).

Le 15, seul le mistral s'illustre encore : $86 \mathrm{~km} / \mathrm{h}$ à Valence-Chabeuil (Drôme).

De puissantes bourrasques sont associées au passage de deux limites les 19 et $20: 94 \mathrm{~km} / \mathrm{h}$ à Mâcon (Sâ̂ne-etLoire) sous orage, $94 \mathrm{~km} / \mathrm{h}$ à Meythet (Haute-Savoie), $104 \mathrm{~km} / \mathrm{h}$ à Chambéry (Savoie) et $108 \mathrm{~km} / \mathrm{h}$ à Riorges (Loire) le 19 ; $94 \mathrm{~km} / \mathrm{h}$ à Ploumanach (Côtesd'Armor), $122 \mathrm{~km} / \mathrm{h}$ au Cap-Corse (Haute-Corse) et $130 \mathrm{~km} / \mathrm{h}$ à Thonon (Haute-Savoie) le 20.

Un net renforcement du vent à l'arrière d'une limite intervient le 21 autour de la Méditerranée et s'essouffle le 22 : $112 \mathrm{~km} / \mathrm{h}$ à Caunes-Minervois (Aude) le $21 ; 83 \mathrm{~km} / \mathrm{h}$ à Salon-de-Provence (Bouches-du-Rhône) le 22.

Le 22, le passage de la queue d'un front chaud provoque également quelques rafales sur l'extrême nord de la France : $90 \mathrm{~km} / \mathrm{h}$ à Dunkerque (Nord).

Un système perturbé génère quelques coups de vent, sur l'Est le 24, le pourtour méditerranéen les 25 et $26: 90 \mathrm{~km} / \mathrm{h}$ à Goin (Moselle) le $24 ; 119 \mathrm{~km} / \mathrm{h}$ au CapBéar (Pyrénées-Orientales) le 25 ; $97 \mathrm{~km} / \mathrm{h}$ à l'île-du-Levant (Var) le 26.

Plus aucune vitesse supérieure à $80 \mathrm{~km} / \mathrm{h}$ n'est enregistrée jusqu'à la fin du mois. Le nombre de jours avec vent fort atteint un maximum de 22 au CapBéar (Pyrénées-Orientales).

\section{Écart à la normale de la température moyenne} (degrés Celsius)

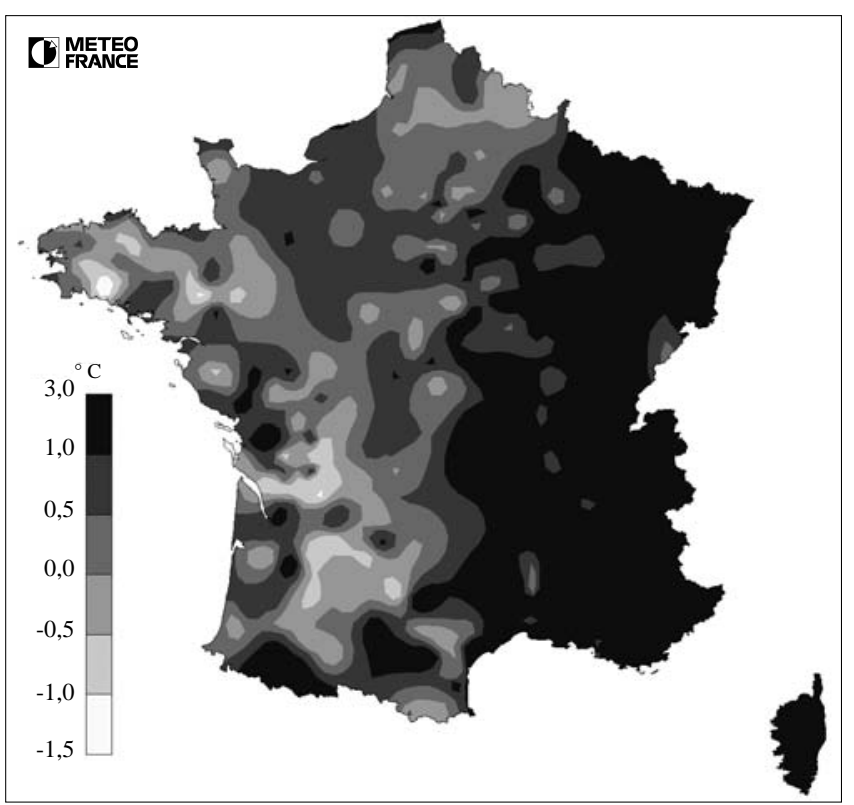

\section{Rapport à la normale des hauteurs de précipitations} (pour cent)

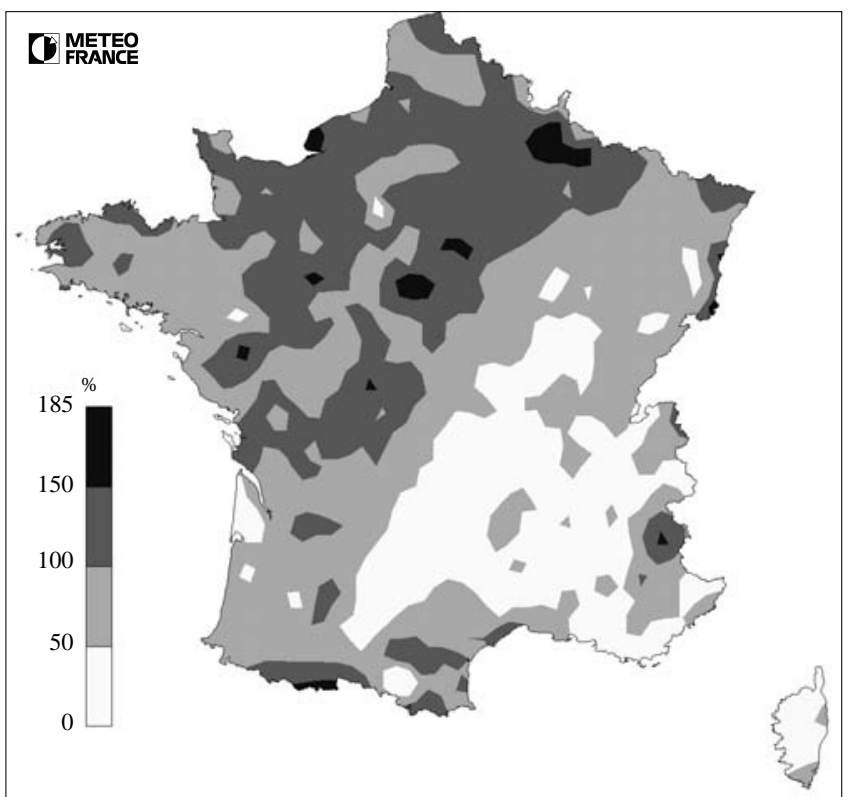

